\title{
Corela
}

Cognition, représentation, langage

HS-30 | 2020

Phonétique, littérature et enseignement du FLE :

théories et recherches

\section{Littérature, phonétique et interactions orales avec les Nouvelles Technologies pour l'apprentissage du Français Langue Étrangère (FLE)}

\section{Mario Tomé Díez}

\section{(2) OpenEdition}

\section{Journals}

Édition électronique

URL : http://journals.openedition.org/corela/11496

DOI : 10.4000/corela. 11496

ISSN : 1638-573X

Éditeur

Cercle linguistique du Centre et de l'Ouest - CerLICO

Référence électronique

Mario Tomé Díez, «Littérature, phonétique et interactions orales avec les Nouvelles Technologies pour l'apprentissage du Français Langue Étrangère (FLE) », Corela [En ligne], HS-30 | 2020, mis en ligne le 01 juillet 2020, consulté le 02 juillet 2020. URL : http://journals.openedition.org/corela/11496 ; DOI https://doi.org/10.4000/corela.11496

Ce document a été généré automatiquement le 2 juillet 2020

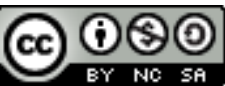

Corela - cognition, représentation, langage est mis à disposition selon les termes de la licence Creative Commons Attribution - Pas d'Utilisation Commerciale - Partage dans les Mêmes Conditions 4.0 International. 


\title{
Littérature, phonétique et interactions orales avec les Nouvelles Technologies pour l'apprentissage du Français Langue Étrangère (FLE)
}

\author{
Mario Tomé Díez
}

\section{Introduction}

1 Dans l'enseignement du Français Langue Étrangère (FLE), différents genres de textes littéraires (extrait d'un ouvrage, poésies, chansons, slogans publicitaires) ont parfois accompagné la pratique pédagogique ou les manuels scolaires. La littérature a occasionnellement fourni des matériaux authentiques à la classe de français, soit pour l'exploitation textuelle, soit par son approche culturelle ou interculturelle (TabakiIona, Proscolli et Forakis, 2010 ; Fiévet, 2013 ; Defays et al., 2014 ; Aubert-Godard, 2015).

2 Mais les apports de la littérature à l'enseignement et à l'apprentissage de la prononciation sont rares. C'est aussi une tendance de la didactique des langues qui néglige souvent l'acquisition des compétences orales, en privilégiant la langue écrite et l'étude de la grammaire et du vocabulaire. Nous croyons que cette tendance pourrait être inversée grâce aux contextes technologiques actuels : ressources et sites Internet, logiciels informatiques pour le traitement de la parole, numérisation et enregistrement audio des textes littéraires, réseaux sociaux et plateformes e-learning pour le développement de la production orale et la correction de la prononciation.

Depuis des années, nous poursuivons des travaux dans cette direction dans le dispositif de formation Campus Virtuel FLE à l'université de León (Espagne $\left.{ }^{1}\right)$. Dans le cadre de cet article, nous voudrions proposer quelques pistes sur les potentiels pédagogiques des nouvelles technologies, ainsi que sur leur application à l'enseignement/apprentissage 
de la prononciation en conjonction avec la littérature. Nous aborderons par la suite les implications didactiques des domaines suivants :

4 1. Une base de données audiovisuelle en ligne sur la littérature française : le projet Littérature Française Multimédia FLE² ;

2. Des tâches sur la littérature (textes, œuvres, auteurs) pour l'acquisition des compétences orales ;

3. Un corpus des productions orales des apprenants de FLE hispanophones : Le projet Oral FLE Prononciation ${ }^{3}$;

4. Des techniques et des méthodes de correction de la prononciation à l'aide des médias sociaux (weblogs, podcasts, réseaux sociaux).

\section{La base de données Littérature Française Multimédia FLE}

5 Dans le cadre du projet FLENET (Français langue étrangère et Internet), l'Université de León ${ }^{4}$ propose, depuis 1999, une base de données audio et vidéo à destination des étudiants, enseignants et chercheurs en FLE. Cette audiovidéothèque en ligne regroupe un ensemble varié de ressources (voir Figure 1):

6 - Des vidéos consacrées aux grands auteurs de la langue française ;

7 - Des audio livres sur les ouvrages de l'histoire de la littérature ;

8 - Des conférences, cours ou séminaires sur la vie ou l'œuvre des écrivains ;

9 - Des émissions radio consacrées à la littérature (interviews, dossiers, magazines) ;

10 - Des livres en ligne ou des extraits des œuvres littéraires ;

11 - Des tâches multimédia pour la découverte ou l'exploitation pédagogique ;

12 - Des sites Web, bibliographies et dossiers sur les œuvres ou les auteurs.

13 Plus d'une centaine d'auteurs sont répertoriés au sein de cette base de données. Un index des noms permet de se retrouver plus rapidement dans le corpus proposé. Des entrées sont également créées pour certains genres et périodes spécifiques de l'Histoire littéraire : Moyen Âge, $\mathrm{xx}^{\mathrm{e}}$ siècle, $\mathrm{xxI}^{\mathrm{e}}$ siècle, romantisme, surréalisme...

14 Les objectifs de ce vaste projet, qui est actualisé périodiquement, sont les suivants :

15 - Privilégier la compréhension orale (CO) pour l'approche des œuvres ou des auteurs ;

16 - Fournir aux enseignants un fonds sonore pour la classe de FLE ou de littérature ;

17 - Favoriser chez les étudiants la découverte et le contact avec la littérature ;

18 - Proposer des tâches pour l'acquisition des compétences orales chez les apprenants de FLE ;

19 - Élaborer une histoire audiovisuelle de la littérature française ou francophone. 


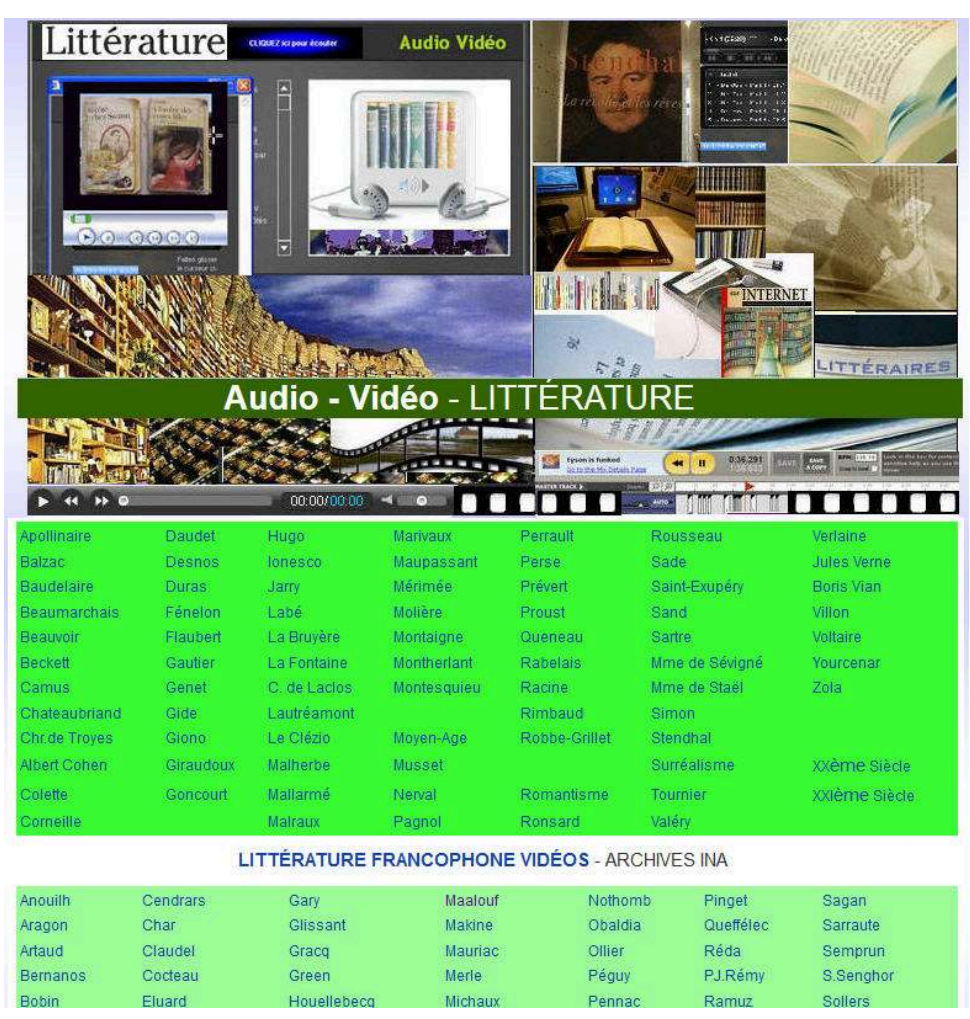

Figure 1 : Site Web de la base de données Littérature Française Multimédia FLE

\section{Des tâches sur la littérature pour l'acquisition des compétences orales}

20 Dans le dispositif Campus Virtuel FLE, les tâches pédagogiques constituent des éléments essentiels pour l'enseignement/apprentissage de la prononciation, et spécialement pour le développement des compétences orales, ainsi que pour la correction de la prononciation. Le modèle de tâche pédagogique (Tomé, 2006) est composé généralement des éléments suivants :

21 - Instructions claires et organisées par étapes ;

22 - Ressources web (textes, sites et matériels audiovisuels) ;

23 - Séquences audio ou vidéo en relation avec la compréhension orale ;

24 - Production orale avec plusieurs modalités d'enregistrement: podcasts (AudioBoo, Soundcloud), magnétophone du PC ou du téléphone portable ;

25 - Pistes, informations ou outils pour accompagner et aider l'apprenant.

Exemple :

Tâche de production orale (TPO) : Le Petit Prince ${ }^{5}$ sur le réseau social Ning (Échanges

Campus FLE Éducation)

Tâche de production orale : Le Petit Prince

Instructions :

Étape 1 : cliquez sur l'image pour regarder la vidéo, puis répondez aux questions.

- Qui est le Petit Prince?

- Que demande-t-il de dessiner?

- Quels sont les autres personnages du récit? 
Étape 2 : Production orale : Enregistrez vos réponses à l'aide de :

Modalités : Podcast - Magnétophone PC - Téléphone portable

Étape 3 : Déposez les réponses et votre enregistrement en utilisant la fonction :

+ Ajouter un nouveau billet à mon blog.

Étape 2 : Production orale : Enregistrez vos réponses à l'aide de :

Modalités : Podcast - Magnétophone PC - Téléphone portable

Étape 3 : Déposez les réponses et votre enregistrement en utilisant la fonction :

+ Ajouter un nouveau billet à mon blog.

Indiquez le lien audio ou déposez le fichier mp3 en utilisant la fonction

« Télécharger un fichier ».

Si vous avez des difficultés, des questions ou des doutes vous pouvez utiliser la

fonction :

Ajouter un commentaire, en bas de ce message.

Pistes et outils complémentaires :

Consultez la rubrique: Saint-Exupéry Littérature Multimédia FLE <http://

flenet.unileon.es/docauteurs2.html\#SaintExupery>.

Audio livre : Le Petit Prince

Livre en ligne : Le petit Prince

Outils : Dictionnaires, correcteurs

Grammaire audiovisuelle FLE : <http://flenet.unileon.es/grammairecours.htm>.

Lexique audiovisuel FLE : <http://flenet.unileon.es/lexiquecours.htm>.

Phonétique française FLE : <http://flenet.unileon.es/phon/indexphon.html>.

\section{Echanges Campus FLE Education}

Communication - Collaboration - Projets interculturels

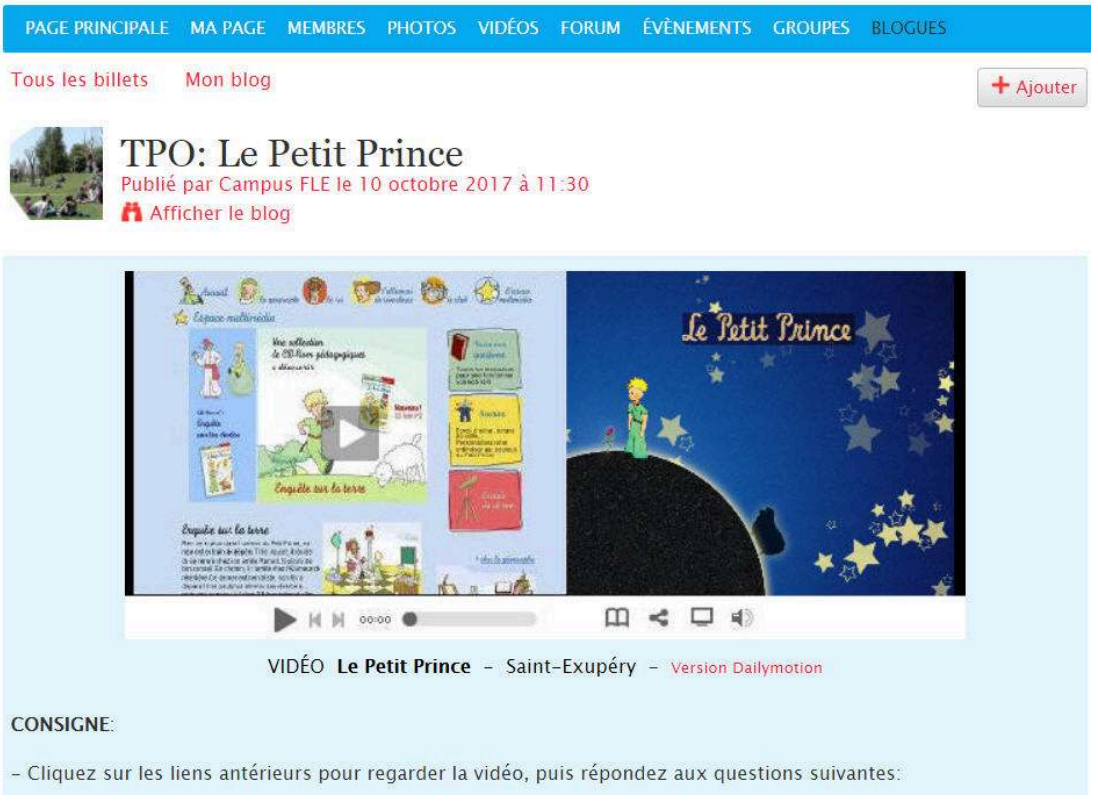

Figure 2 : Site Web de la tâche de production orale : Le Petit Prince

Pour notre recherche, nous avons élaboré et essayé différentes tâches pédagogiques pour le développement des compétences orales, et en relation avec l'apprentissage de la prononciation nous nous sommes centrés sur les objectifs suivants (Tomé 2007; 2009 ; $2011 ; 2016)$ :

- Développer les compétences de production et compréhension orales chez les apprenants ; 
- Favoriser la correction de la prononciation entre apprenants, tuteurs et enseignants ;

- Faciliter des contextes réels de communication et de collaboration.

Nous avons distingué quatre modalités de tâches (Tomé, 2006) : a.compréhension orale; b. production orale ; c. correction de la prononciation; d. interculturelles. Nous présentons quelques exemples en relation avec la littérature, que l'on peut consulter en ligne sur les sites signalés dans les notes :

A. Tâches de compréhension orale (CO)

- Dictées multimédia autocorrectives ${ }^{6}$. Des textes littéraires en version audio avec la transcription pour la correction de la dictée : Daudet, « La chèvre de M. Seguin »; SaintExupéry, Terres des hommes ; Giraudoux, La guerre de Troie n'aura pas lieu ; Barrès, Greco ou le Secret de Tolède ; Proust, Du côté de chez Swann.

- Textes Audio - Flenet ${ }^{7}$. Des extraits de romans en version audio avec ses textes: Camus, L'étranger ; Perrault, Le Petit Poucet ; Maupassant, Le Horla ; Vian, L'écume des jours ; Stendhal, Le Rouge et le Noir ; Zola, Germinal ; Chateaubriand, René; B. de Saint-Pierre, Paul et Virginie.

- Vidéo Activités Pédagogiques ${ }^{8}$. Des courts documentaires en version vidéo sur des aspects de la culture et la littérature françaises : Stendhal et Grenoble, Le Petit Prince, Baudelaire («Hymne à la beauté »), Corneille Actualités, Stendhal Exposition, Nothomb Mystères, Découvertes Astérix et Tintin, La Marseillaise - Compréhension Orale.

B. Tâches de production orale (PO)

32 - Exercices de répétition ou sensibilisation aux phonèmes, mots ou chansons ${ }^{9}$ : des onomatopées, des cris, des virelangues ou des chansons pour l'exploitation et le renforcement des phonèmes problématiques: Jeux, Cris et Chansons; Les étudiants chantent des chansons ${ }^{10}$.

- Lecture de textes littéraires. Les Textes Audio - Flenet du chapitre antérieur ont été conçus pour cette intention, ainsi que la vidéo activité Baudelaire: "Hymne à la beauté » ou la Tâche PO et Production écrite (PE) : Visite Bibliothèque virtuelle ${ }^{11}$.

- Tâches multimédia pour la production orale. Elles présentent les caractéristiques et objectifs que nous avons indiqués plus haut, en suivant le modèle de la TPO (Le Petit Prince) : À la découverte de la littérature française; CybeRallye FLE : Jules Verne; À la découverte de la chanson française; Vendredi ou la vie sauvage - Tâches orales ${ }^{12}$.

C. Tâches de correction de la prononciation

Celles-ci seront abordées dans les derniers chapitres :

- Autocorrections individuelles : échauffement vocal et Prononciation FLE ;

- Corrections collaboratives entre étudiants : TPO (Le Petit Prince);

- Jeux, dramatisations, créations: correction collaborative Prononciation-Vidéo Tâches.

D. Tâches interculturelles

Plusieurs tâches multimédia pour la production orale ont comme objectif la découverte d'un aspect culturel (musées, monuments, art, cinéma) ou littéraire comme quelques exemples précédents. 


\section{Les médias sociaux pour la production orale}

38 L'application et la sélection de différents médias sociaux dans nos travaux se sont appuyées sur les critères suivants :

a. Intégration d'un magnétophone pour l'enregistrement des séquences audio.

b. Développement de la production orale et de la correction de la prononciation.

41 c. Potentiel de collaboration entre pairs (écoute mutuelle, commentaires, corrections ou créations en commun).

42 Nous avons analysé le rôle de ces outils dans de diverses publications (Tomé 2007; $2009 ; 2011 ; 2015 ; 2016)$. Les plus décisifs pour la production orale, ainsi que pour l'élaboration du corpus Oral FLE Prononciation ont été les suivants :

43 - Podomatic est un service en ligne qui permet de créer des podcasts audio et vidéo. Il dispose d'un magnétophone web très utile pour enregistrer des messages sonores. Nous avons créé des audio blogs de la classe, comme l'Audio Carnet FLE 2005-06, où l'on rassemblait les tâches pédagogiques et les productions orales des apprenants de FLE.

44 - AudioBoo, Soundcloud, Twaud.io et Jamglue étaient les espaces d'enregistrement ainsi que les dossiers ou cahiers sonores des étudiants. L'interface dynamique et simple permettait le tutorat de l'enseignant et facilitait la création des fichiers audio par les apprenants (voir Figure 4).

45 - UStream est une communauté de live broadcasting en vidéo (émission en direct) qui rend possible la retransmission de séquences vidéo à partir d'une simple connexion de webcam, ce qui permet de faire des webconférences ou des émissions de télévision. Avec cet outil les étudiants ont réalisé plusieurs tâches de correction de la prononciation en collaboration.

46 - Ning est une plate-forme de réseaux sociaux qui offre un grand potentiel pour la collaboration entre étudiants. Elle a servi de support pour des tâches de production orale et de collaboration entre étudiants, ainsi que pour les échanges entre des tuteurs et des apprenants dans des projets de télécollaboration.

\section{Le corpus Oral FLE Prononciation}

47 Les productions orales des apprenants de FLE ont été présentées et analysées dans des publications précédentes (Tomé $2009 ; 2011 ; 2015 ; 2016$ ). Depuis 2005, on a mis en place une base de données audiovisuelle sur ces matériaux sonores qui est disponible en ligne dans le Projet Oral FLE Prononciation ${ }^{13}$. Les objectifs fondamentaux de ce corpus oral sont les suivants :

48 - Constituer un fonds sonore à partir des enregistrements audio et des productions orales des étudiants de FLE déposés dans les podcasts, weblogs, réseaux sociaux et plateforme de formation (Moodle) ;

49 - Classifier et analyser ces productions orales dans le contexte éducatif de l'enseignement/apprentissage de la prononciation du français langue étrangère ;

50 - Proposer à la communauté scientifique du FLE les résultats et les expériences de ces recherches. 
51 baladodiffusion (weblogs, podcasts, lecteurs multimédia, magnétophones web) ont joué un rôle très important, parce que la plupart des enregistrements audiovisuels ont été réalisés à l'aide de ces médias sociaux. Les premiers enregistrements de la production orale publiés sur Internet, et expérience pionnière dans l'apprentissage des langues, ont été réalisés en octobre 2005 à l'Université de León, par les apprenants d'un cours de FLE ( $2^{a}$ Lengua y su Literatura Francés I), dans le cadre du weblog Audio Carnet FLE 2005-2006 ${ }^{14}$. Quelques auteurs (Belanger, 2005 ; McCarty, 2005) ont déjà remarqué que les premières applications du podcasting pour la compréhension orale ont eu lieu dans un cours d'anglais au Osaka Jogakuin College (Japon), ainsi que les premiers enregistrements audio (sur des iPods) ont eu lieu dans un cours d'espagnol au printemps 2005 à l'Université de Duke (États-Unis). Malheureusement, on ne conserve pas d'archives sonores de ces pratiques historiques.

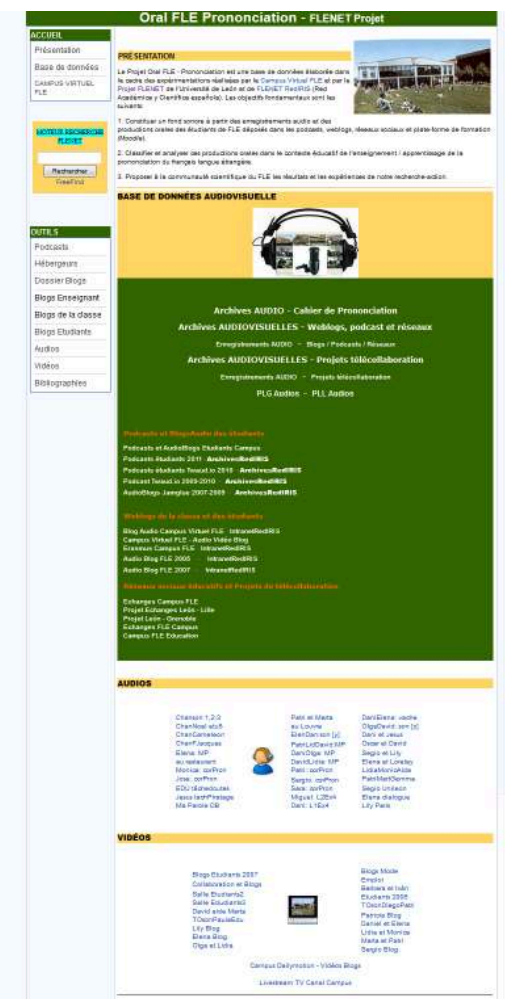

Figure 3 : Site Web du Projet Oral FLE Prononciation

\section{1. Le système des erreurs des apprenants hispanophones}

Le corpus Oral FLE Prononciation peut être considéré comme la première base de données orale sur le système des erreurs de prononciation des apprenants espagnols. Même si des auteurs avaient déjà fait référence aux principales erreurs de prononciation des hispanophones (Léon et Léon, 1964 ; Companys, 1966 ; Callamand, 1981 ; Tomé, 1994), c'est la première fois que ce système d'erreurs est fondé sur un corpus oral d'enregistrements audiovisuels.

Dans le système vocalique, nous croyons qu'il est très utile d'appliquer une simplification pédagogique, spécialement avec les apprenants intermédiaires (niveaux B1 et B2). Plusieurs auteurs avaient déjà suggéré cette démarche pédagogique : Léon et 
Léon (1964), Companys (1966), Tomé (1994), Wioland (1997), Lauret (2007), Weber (2013), pour ne citer qu'eux. Nous avons ainsi travaillé avec des archiphonèmes qui impliquent la neutralisation ou disparition de certaines oppositions vocaliques, afin de surmonter les erreurs, faciliter l'articulation et l'apprentissage de certains sons et favoriser l'acquisition d'une prononciation correcte chez les apprenants hispanophones. Nous avons distingué sept sons fondamentaux : l'archiphonème /A/ qui remplace l'opposition $[\mathrm{a}]-[\mathrm{a}]$; l'archiphonème /E/ face à l'opposition $[\varepsilon]-[\mathrm{e}]$; l'archiphonème /OE/ qui suppose la neutralisation des phonèmes [œ]-[ə]-[ø]; l'archiphonème /O/ à la place de l'opposition [o]-[0]; l'archiphonème /Ẽ/ qui implique la suppression de l'opposition $[\tilde{\varepsilon}]-[\tilde{e}]$; et finalement le reste des phonèmes

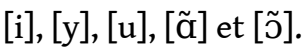

54 Les productions orales et enregistrements audio des apprenants de FLE nous ont permis d'établir un système d'erreurs qui est constitué par dix phonèmes et deux archiphonèmes. Ce sont par ordre de difficulté et de priorité dans la correction les

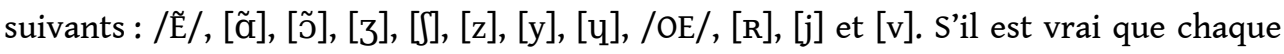
apprenant présente son propre système d'erreurs, les résultats obtenus dans la base de données soulignaient les erreurs les plus problématiques et prioritaires. Les aspects suivants sont aussi déterminants dans le processus de correction phonétique :

55 - Importance de l'effort articulatoire et du renforcement pour dépasser les erreurs ;

- Présence et application de différentes techniques de correction chez les apprenants;

- Indices d'amélioration dans l'acquisition de la prononciation: répétitions, échauffements, auto-corrections, théâtralisations et collaborations.

Nous présenterons plus loin plusieurs exemples sur les techniques et méthodes de correction de la prononciation. Finalement, nous résumons les composantes du corpus oral en relation avec la littérature ${ }^{15}$ :

57 - Phonèmes et chansons: on a sélectionné des comptines ou des poèmes ${ }^{16}$ qui favorisaient la sensibilisation (compréhension ou production orales) face aux principales difficultés articulatoires des apprenants, comme par exemple : [y] « Loup y es-tu? »; /OE/ «Adieu, adieu capitaine »; / Ẽ/ «Petit pingouin »; [z] « La Javanaise »; [3] «En passant les Pyrénées». Les étudiants ont fait des enregistrements récitant généralement ces chansons et parfois appliquant certaines techniques de correction.

- Lecture de textes littéraires à haute voix : à partir de différentes tâches ou de Textes Audio - Flenet les étudiants procédaient à une préparation du texte qu'ils devaient enregistrer, ce qui impliquait parfois des entraînements/échauffements articulatoires, des répétitions ou des corrections phonétiques collaboratives.

- Méthodes et techniques de correction de la prononciation qui ont été recueillis dans certains enregistrements.

\section{Les techniques et méthodes de correction de la prononciation}

Plusieurs recherches ont déjà abordé ou défini différents types de correction de la prononciation, que ce soit de la part de l'enseignant ou des apprenants: explicit correction, recasts, clarification requests, metalinguistic feedback, elicitation, repetition, reinforcement (Lyster et Ranta, 1997 ; Neri et al., 2002 ; Engwall, 2006 ; Engwall et Bälter, 2007). Abry et Veldeman-Abry (2007) et, spécialement, Lauret (2007) ont recueilli de 
nombreuses tâches et méthodes de correction phonétique : échauffement, imitation, répétition, jeux de rôles, échauffement, gestuelle du corps, articulation exagérée, jeu théâtral, explications acoustiques ou articulatoires.

Dans des publications précédentes nous avons déjà fait référence aux différentes modalités et techniques de correction de la prononciation à l'aide des médias sociaux : weblogs, podcasts, réseaux sociaux (Tomé 2009; 2015; 2016). Nous présenterons quelques exemples qui font partie du corpus Oral FLE Prononciation ou de la section yLitteraturePhon en remarquant leur intérêt et leurs conséquences pédagogiques pour l'amélioration de la prononciation.

\section{1. Échauffement vocal}

Cet entraînement préalable à la production orale qui, occasionnellement, peut être recueilli dans certains enregistrements audio implique une auto-conscience des difficultés articulatoires. L'apprenant s'exerce à la maîtrise d'un trait phonique (arrondissement, nasalisation, articulation sonore, fricative, palatale ou uvulaire) afin de pouvoir ainsi surmonter ses erreurs ou difficultés. Cette pratique peut se centrer sur la répétition d'un phonème ou bien s'accompagner d'un cri, d'une onomatopée ou d'une chanson, etc.

Transcription 1: L'étudiante Maria enregistre des échauffements sur les voyelles nasales de trois chansons ${ }^{17}$ :

Maria : Les voyelles nasales. $\mathrm{O}, \mathrm{O}, \mathrm{O}$, on, on on. « Les sanglots longs / des violons / de l'automne / bercent mon cœur / d'une langueur monotone" (Paul Verlaine). A, A,

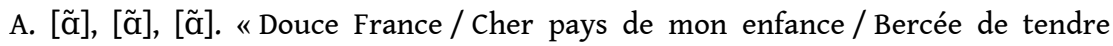
insouciance» (Charles Trenet). E, E, E. /Ẽ/, /Ẽ/, /Ẽ/. «Petit pingouin / tous les matins / tu sautes d'un pied / sur l'autre pied / pour te réveiller» (comptine). (voir fichier audio: Maria_echauff_3chNasales.m4a, sur un poème, une chanson et une comptine).

\subsection{Effort ou renforcement articulatoire}

La pratique de l'effort articulatoire est un facteur fondamental pour l'éveil de la conscience phonologique face à l'erreur. L'apprenant doit marquer ou même exagérer l'articulation d'un phonème, en ayant recours à différentes méthodes: oppositions phonologiques $([\mathrm{i}]-[\mathrm{y}]-[\mathrm{u}]$; voyelle orale ou nasale; consonne sourde ou sonore);

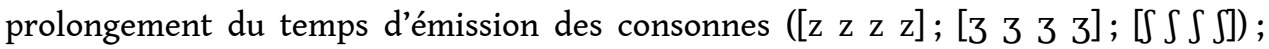
physiologie de l'appareil phonatoire (arrondissement des lèvres; cavité nasale; vibration des cordes vocales ou de la luette) ou simple répétition d'un son.

Transcription 2 : L'étudiante Sandra lit une comptine sur le phonème [y], en marquant et allongeant l'articulation de celui-ci ${ }^{18}$ :

Sandra : Effort articulatoire. Le son [y]. « Loup y es-tu ? / Que fais-tu ? / M’ entends-

tu?» (voir fichier audio Sandra_EAchLoup.m4a, sur une comptine).

\subsection{Autocorrection}

64 L'apprenant doute, repère une possible erreur et se corrige lui-même. Ce type de correction met en relief une importante attitude pédagogique parce qu'elle implique une réflexion de l'étudiant sur son propre processus d'apprentissage. L'autocorrection 
peut être spontanée ou théâtralisée, et parfois difficile de différencier dans les enregistrements audio des apprenants.

Transcription 3: l'apprenant s'autocorrige quand il doit prononcer dans le mot « chandelle » la consonne []]. Conscient d'une possible erreur il dit « non » et, ensuite, il reprend une articulation renforcée et exagérée ${ }^{19}$ :

Étudiant: Ma chandelle, sssss, $\mathrm{IOJ}$; non $\mathrm{IOW}$, ma chandelle est morte (voir fichier audio 2voix_Auclairlune.mp3, autour de la lecture du poème/chanson « Au clair de la lune »).

\subsection{Explications linguistiques}

L'enseignant, tuteur ou pair se sert d'un symbole de l'Alphabet Phonétique International, propose la définition d'un phonème ou introduit une notion de phonétique articulatoire (appareil phonatoire, position des lèvres) ou de phonologie (opposition, sonorisation, arrondissement).

67 Transcription 4: La tutrice Valérie corrige la production orale d'un étudiant en utilisant les symboles ou correspondances écrites des phonèmes ${ }^{20}$ :

Valérie : décoiffée : «oi » se prononce [ wa ] et « ée » se prononce / E /; ne put : on prononce $[y]$; suis, soleil : on prononce $[s] ;$ je : on prononce [graphie $" \mathrm{j} »]$ (voir commentaire écrit dans un réseau social sur la tâche : Le Petit Prince).

\subsection{Correction collaborative}

Deux (ou plus) apprenants travaillent ensemble et se corrigent mutuellement. L'un d'entre eux prend le rôle de l'enseignant ou tuteur en relevant systématiquement les erreurs de l'autre. Les corrections collaboratives représentent l'un des principaux enjeux de notre recherche. Nous constatons que les différents types de correction de la prononciation sont particulièrement utiles lorsqu'ils se réalisent en collaboration car ils favorisent la motivation, l'auto-conscience des erreurs, l'amélioration des difficultés articulatoires et la mise en place de situations réelles de communication.

Transcription 5 : trois étudiants travaillent ensemble la lecture à haute voix des paroles d'une chanson. Leur enregistrement met en évidence les efforts et renforcements, les répétitions et les corrections mutuelles ${ }^{21}$ :

Étudiant 1 : Bonjour ma cousine.

Étudiante 2 : Bonjour mon cousin germain.

Étudiante 3 : On m'a dit que vous m'aimiez.

Étudiant 1 : Est-ce bien la vérité ?

Étudiante $2: \mathrm{F}$. Je ne m'en soucie guère.

Étudiante 3 : Passez par ici et moi par là.

Étudiant 1: Au revoir ma cousine on se reverra (voir fichier audio 3voix_BonjourMaCousine.mp3, autour de la lecture du poème/chanson « Bonjour ma cousine »).

\subsection{Répétition}

70 C'est une pratique classique dans l'enseignement des langues, laquelle suppose généralement un dialogue ou interaction entre personnes. L'enseignant, tuteur ou pair, propose la répétition d'un phonème, mot ou phrase que l'apprenant doit prononcer à une ou à plusieurs reprises. Les répétitions font souvent partie des techniques 
antérieures de correction et leur présence dans les enregistrements audio constitue un important indice d'amélioration de la prononciation.

71 Transcription 6: dans un enregistrement collaboratif deux étudiantes préparent et chantent une chanson. L'une propose à l'autre la répétition de quelques mots ${ }^{22}$ :

Étudiante 1 : Au clair de la lune.

Étudiante 2 : Au clair de la lune.

Étudiante $1:$ lune.

Étudiante $2:$ lune.

Étudiante 1 : mon ami Pierrot.

Étudiante 2 : mon ami Pierrot.

Étudiante 1 : Prête-moi ta plume.

Étudiante 2 : Prête-moi ta plume.

Étudiante 1 : plume.

Étudiante 2 : plume.

Étudiante 1 : pour écrire un mot.

Étudiante 2 : pour écrire un mot.

Étudiante 1 : écrire

Étudiante 2 : écrire

Étudiante 1 : un mot.

Étudiante 2: un mot (voir Podcast AudioBoo de l'étudiant, fichier

2voix_CCauclairlune.mp3, sur la chanson « Au clair de la lune »).

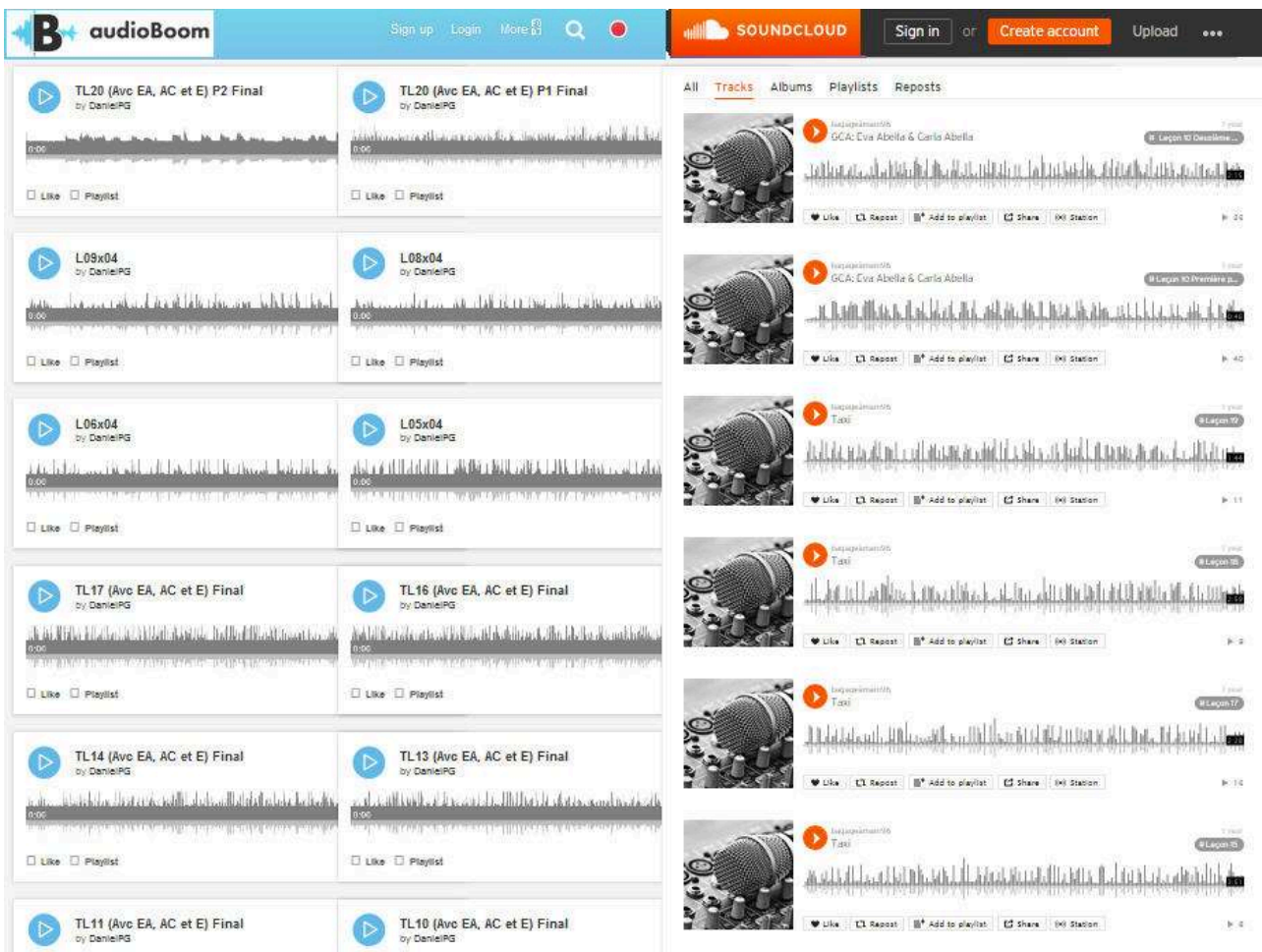

Figure 4 : productions orales des étudiants sur AudioBoom et SoundCloud

\section{Perspectives}

Il faudra poursuivre de nouvelles recherches sur les applications des médias sociaux en classe de français. Les différents domaines de la littérature pourraient fournir des matériaux didactiques intéressants pour l'apprentissage de la prononciation et la correction phonétique. La base de données Littérature Multimédia FLE devrait s'actualiser 
et s'enrichir avec de nouvelles ressources audiovisuelles. Nous croyons qu'il serait possible de constituer une vaste audiovidéothèque en ligne de l'histoire de la littérature française, complémentaire de l'immense patrimoine écrit disponible actuellement sur le web.

L'élaboration de nouvelles tâches pédagogiques sur la littérature devrait être prioritaire dans cette base de données, et spécialement les tâches pour la production orale et pour l'apprentissage de la prononciation. Les enseignants devraient conjuguer les médias sociaux avec des tâches efficaces et motivantes qui actualiseraient des situations réelles de communication et de collaboration chez les étudiants. Les futures transformations d'Internet avec l'arrivée de nouveaux outils (réseaux et médias sociaux, développement de la communication et de la technologie sur la parole) et des espaces d'interaction permettront d'intégrer des tâches décisives pour l'acquisition des compétences linguistiques et interculturelles.

Le projet Oral FLE Prononciation devrait se perfectionner dans plusieurs directions: renouveler et agrandir la base de données actuelle; organiser de nouvelles catégories sur le système d'erreurs des apprenants espagnols de FLE ; établir une typologie sur les méthodes de correction phonétique pratiquées par les enseignants, tuteurs et étudiants; analyser et évaluer la fréquence des erreurs de prononciation; compléter les transcriptions orthographiques des fichiers audio et appliquer des logiciels de traitement de la parole.

\section{BIBLIOGRAPHIE}

Abry D. et Veldeman-Abry J., 2007, La phonétique : audition, prononciation, correction, Paris, CLE International, coll. « Techniques et pratiques de classe ».

Belanger Y., 2005, Duke University iPod first year experience final evaluation report, Durham, Duke University.

Callamand M., 1981, Méthodologie de l'enseignement de la prononciation : organisation de la matière phonique du français et correction phonétique, Paris, CLE International.

Companys E., 1966, Phonétique française pour hispanophones, Paris, Hachette/Larousse.

Defays J.-M., Delbart A.-R., Hammami S. et Saenen F., 2014, La littérature en FLE :état des lieux et nouvelles perspectives, Paris, Hachette FLE, coll. « $\mathrm{f} »$.

Engwall O., 2006, « Feedback strategies of human and virtual tutors in pronunciation training », TMH-QPSR, no 48, p. 11-34. Disponible sur : <http://www.speech.kth.se/prod/publications/files/ qpsr/2006/2006_48_1_011-034.pdf>.

Engwall O. et Balter O., 2007, « Pronunciation Feedback from Real and Virtual Language Teachers ", Computer Assisted Language Learning, vol. 20, no 3, p. 235-262.

Fiévet M., 2013, Littérature en classe de FLE, Paris, CLE International, coll. « Techniques et pratiques de classe ». 
Godard A. (dir.), 2015, La littérature dans l'enseignement du FLE, Paris, Didier, coll. « Langues et didactique ».

Lauret B., 2007, Enseigner la prononciation du français : questions et outils, Paris : Hachette, coll. « $\mathrm{f} »$.

Léon P. et Léon M., 1964, Introduction à la phonétique corrective, Paris, Hachette/Larousse.

Lyster R. et Ranta L., 1997, « Corrective feedback and learner uptake », Studies in Second Language Acquisition, no 19, p. 37-66. Disponible sur : <http://people.mcgill.ca/files/roy.lyster/ Lyster_Ranta1997_SSLA.pdf>.

McCarty S., 2005, « Spoken Internet To Go : Popularization through Podcasting », The JALT CALL Journal, vol. 1, no 2, p. 67-74. Disponible sur : <http://journal.jaltcall.org/articles/

1_2_McCarty.pdf>.

Neri A., Cucchiarini C., Strik H. et Boves L., 2002, « The pedagogy-technology interface in computer-assisted pronunciation training ", Computer-Assisted Language Learning, vol. 15, no 5, p. 441-467. Disponible sur : <http://lands.let.kun.nl/literature/neri.2002.3.pdf>.

Tabaki-Iona F., Proscolli A. et Forakis K. (dir.), 2010, La place de la littérature dans l'enseignement du FLE. Actes du Colloque international des 4 et 5 juin 2009, Athènes, Université nationale et capodistrienne d'Athènes. Disponible sur : <http://fr.frl.uoa.gr/fileadmin/frl.uoa.gr/uploads/ sinedria/Actes_Litterature\%20FLE_2009.pdf>.

Tomé M., 1994, Fonética francesa para uso de estudiantes españoles, León, Universidad de Léon. -, 2006, « L'enseignant de FLE et les ressources Internet ", Çédille. Revista de estudios franceses, no 2, p. 114-133. Disponible sur : <http://cedille.webs.ull.es/dos/tome.pdf>.

-, 2007, « Expériences pédagogiques dans le Campus Virtuel FLE », ÉLA (Études de linguistique appliquée), no 146, p. 179-188. Disponible sur : <https://www.cairn.info/revue-ela-2007-2page-179.htm>.

-, 2009, « Productions orales, weblogs et projet de télécollaboration avec le web 2.0 pour l'enseignement du français (FLE) ", ALSIC (Apprentissage des Langues et Systèmes d' Information et de Communication), vol. 12, p. 90-108. Disponible sur : <http://journals.openedition.org/alsic/1279>.

-, 2011, « Réseaux et médias sociaux sur Internet pour l'apprentissage de la prononciation d'une langue étrangère », International Journal of E-learning \& Distance Education, vol. 25, no 2. Disponible sur : <http://www.ijede.ca/index.php/jde/article/view/724/1261>.

,- 2015 , « Development, analysis and evaluation of oral production in foreign language class with new technologies ", Thélème. Revista Complutense de Estudios Franceses, vol. 30, no 2, p. 255-268. Disponible sur : <http://revistas.ucm.es/index.php/THEL/article/view/48663>.

,- 2016 , «Compétences orales et nouvelles technologies dans un cours de français langue étrangère ", Çédille. Revista de estudios franceses, no 12, p. 387-401. Disponible sur : <http:// cedille.webs.ull.es/12/20tome.pdf>.

Weber C., 2013, Pour une didactique de l'oralité. Enseigner le français tel qu'il est parlé, Paris, Didier, coll. « Langues \& didactique ».

Wioland F., 1991, Prononcer les mots du français : des sons et des rythmes, Paris, Hachette FLE, coll. « F/autoformation ». 


\section{NOTES}

1. Campus Virtuel FLE, Universidad de León : <http://flenet.unileon.es/BSCWprojet/index.html>.

2. Le site principal de la base de données Littérature Française Multimédia FLE, < http:// flenet.unileon.es/docauteurs.html>, ainsi que Littérature Audiovisuelle, <http:// flenet.unileon.es/culturecours.html>, et Littérature Francophone Vidéos <http:// flenet.unileon.es/docauteursVideo.html>.

3. Oral FLE Prononciation : <http://flenet.rediris.es/OralFLEprojet/index.htm>.

4. FLENET (Français Langue Étrangère et Internet) : <http://flenet.unileon.es/>.

5. La tâche de production orale: Le Petit Prince est disponible sur le réseau social Échanges Campus FLE Education (< http://campusfle.ning.com/profiles/blogs/tpo-lepetit-prince>), ainsi que sur le weblog Campus Virtuel FLE (<https://goo.gl/i296CS>).

6. Dictées multimédia autocorrectives : <http://flenet.unileon.es/phon/dicteacti.html>.

7. Textes Audio-Flenet : <http://flenet.unileon.es/courstourdumonde/textesaudio/ textes.html>.

8. Vidéo Activités Pédagogiques: <http://flenet.rediris.es/actipedago.html\#tachesVideo> et <http://flenet.unileon.es/xapliex1.htm\#ActiPVideo> ; «La Marseillaise »-Compréhension orale : $<$ http://campusfle.ning.com/profiles/blogs/la-marseillaise-compr-hension-orale>.

9. Chansons pour les phonèmes FLE: <http://flenet.unileon.es/courstourdumonde/ carnetph.htm\#PhonChansons>.

10. Jeux, Cris et Chansons (<https://goo.gl/VoF5CG>) ; Les étudiants chantent des chansons : $<$ https://goo.gl/F52Q3Y>.

11. Tâche PO et PE : Visite Bibliothèque virtuelle (<http://campusfle.ning.com/profiles/blogs/ tache-po-et-pe-visite>).

12. TPO : À la découverte de la littérature française (<http://campusfle.ning.com/profiles/blogs/ tpo-a-la-decouverte-de-la>); CybeRallye FLE: Jules Verne (<http://flenet.rediris.es/acti/ cyberallyeJVerne.html>); À la découverte de la chanson française (<http:// erasmus07.canalblog.com/archives/2006/11/02/3060793.html>); Vendredi ou la vie sauvagetâches orales : <http://campusfle.ning.com/profiles/blogs/vendredi-ou-la-vie-sauvage-t-chesorales>.

13. Projet Oral FLE Prononciation : <http://flenet.rediris.es/OralFLEprojet/index.htm>.

14. Audio Carnet FLE 2005-2006 : <https://www.podomatic.com/podcasts/campus6>. Ces podcasts historiques sont disponibles sur: Presentacion audio Elena-Loreley (<https:// www.podomatic.com/podcasts/campus6/episodes/2005-10-25T09_54_39-07_00>) et Bonjour de Dani et Jesus (<http://flenet.rediris.es/weblogs/PODOMATIC_Audioblogs/ PodomaticFLE2005_DaniJesus.html>).

15. Les productions orales en relation avec la littérature sont dans le dossier yLitteraturePhon: $<$ http://flenet.rediris.es/OralFLEprojet/yLitteraturePhon>.

16. Chansons et poèmes audio: <http://flenet.unileon.es/phon/jeux/index.html\#chansons>.

17. Pour écouter l'enregistrement audio: <http://flenet.rediris.es/OralFLEprojet/ yLitteraturePhon/_ChanPhon_CInter/Maria_echauff_3chNasales.m4a>.

18. Pour écouter l'enregistrement audio: <http://flenet.rediris.es/OralFLEprojet/ yLitteraturePhon/_ChanPhon_CInter/Sandra_EAchLoup.m4a>.

19. Pour écouter l'enregistrement audio: <http://flenet.rediris.es/OralFLEprojet/ yLitteraturePhon/_ChanPhon_CInter/2voix_Auclairlune.mp3>.

20. Pour consulter le commentaire dans Tâche TPO (Le Petit Prince) : <http://campusfle.ning.com/ profiles/blogs/tpo-le-petit-prince>.

21. Pour écouter l'enregistrement audio: <http://flenet.rediris.es/OralFLEprojet/ yLitteraturePhon/_ChanPhon_CInter/3voix_BonjourMaCousine.mp3>. 
22. Pour écouter l'enregistrement audio: <http://flenet.rediris.es/OralFLEprojet/ yLitteraturePhon/Irene_chansons/2voix_CCauclairlune.mp3>.

\section{RÉSUMÉS}

Des revues et des ouvrages spécialisés ont préconisé l'exploitation des textes littéraires dans l'apprentissage $\mathrm{du}$ français langue étrangère (FLE). Mais les recherches et les pratiques pédagogiques axées sur les apports de la littérature dans l'enseignement de la prononciation sont rares. En didactique des langues, les enseignants ont tendance à négliger, très souvent, l'acquisition des compétences orales, face à la concurrence de la langue écrite, de la grammaire ou du vocabulaire. Dans cet article, nous présentons les expériences sur le développement de la production orale et la correction de la prononciation en relation avec la littérature dans le dispositif de formation Campus Virtuel FLE à l'Université de León (Espagne). À l'heure du numérique nous aurons recours aux multiples ressources, outils, environnements informatiques et médias sociaux en relation avec les domaines suivants: 1. Le projet Littérature Française Multimédia FLE, une base de données audiovisuelle en ligne sur la littérature française pour l'enseignement et apprentissage du FLE ; 2. Les tâches sur la littérature (textes, œuvres, auteurs) pour l'acquisition des compétences orales ; 3 . Le corpus Oral FLE Prononciation des productions orales des apprenants hispanophones; 4. Les techniques et méthodes de correction de la prononciation à l'aide des médias sociaux (weblogs, podcasts, réseaux sociaux).

Journals and specialized works have advocated the exploitation of literary texts in the learning of French as a foreign language (FLE). But research and pedagogical practices focused on the contributions of literature in the teaching of pronunciation are rare. In language didactics, teachers tend to neglect very often the acquisition of oral skills in the face of competition from written language, grammar or vocabulary. In this article we present the experiments on the development of oral production and pronunciation correction in relation to literature in the training device Virtual Campus FLE at the University of León (Spain). In the digital age we will use multiple resources, tools, environments and social media related to the following areas: 1. The project Literature Française Multimédia FLE, an online audiovisual database on French literature for the teaching and learning of FLE; 2. The tasks on literature (texts, works, authors) for the acquisition of oral skills; 3 . The Oral FLE corpus Pronunciation of oral productions of Spanish-speaking learners; 4 . Techniques and methods of pronunciation correction using social media (weblogs, podcasts, social networks).

\section{INDEX}

Mots-clés : apprentissage de la prononciation, correction phonétique, Français Langue Étrangère (FLE), littérature française.

Keywords : pronunciation learning, phonetic correction, French as a Foreign Language, French literature. 
AUTEUR

MARIO TOMÉ DÍEZ

Universidad de León 